\title{
Asymptotic Solutions of the Elastic Wave Equation and Reflected Waves near Boundaries
}

\section{Hideo Soga}

Faculty of Education, Ibaraki University, Mito Ibaraki, 310 Japan

Received July 6, 1989; in revised form February 27, 1990

\begin{abstract}
In the first half of this paper, we construct asymptotic solutions of linear anisotropic elastic equations. In the latter half, we investigate waves reflected by boundaries for plane incident waves in terms of these solutions. Especially, it is examined whether or not the mode-conversion occurs near points where the incident waves hit the boundaries perpendicularly.
\end{abstract}

\section{Introduction}

Let $\Omega$ be a domain in $\mathbb{R}_{x}^{n}\left(x={ }^{t}\left(x_{1}, \ldots, x_{n}\right), n \geqq 2\right)$ with a $C^{\infty}$ boundary $\partial \Omega$, and consider the elastic wave equation

$$
\left(\partial_{t}^{2}-\sum_{i, j=1}^{n} a_{i j} \partial_{x_{i}} \partial_{x_{j}}\right) u(t, x)=0 \quad \text { in } \quad \mathbb{R} \times \Omega .
$$

Here, $u={ }^{t}\left(u_{1}, \ldots, u_{n}\right)$ is the displacement vector, and $a_{i j}$ are real constant $n \times n$ matrices whose $(p, q)$-components are denoted by $a_{i p j q}$. We assume that $a_{i j}$ satisfy

$$
\begin{aligned}
& a_{i p j q}=a_{p i j q}=a_{j q i p}, \quad i, j, p, q=1,2, \ldots, n, \\
& \sum_{i, p, j, q=1}^{n} a_{i p j q} \varepsilon_{j q} \bar{\varepsilon}_{i p} \geqq \delta \sum_{i, p=1}^{n}\left|\varepsilon_{i p}\right|^{2} \quad \text { for every Hermitian matrices }\left(\varepsilon_{i j}\right), \\
& \sum_{i, j=1}^{n} a_{i j} \xi_{i} \xi_{j} \text { has eigenvalues of constant multiplicity for } \\
& \quad \text { any } \xi={ }^{t}\left(\xi_{1}, \ldots, \xi_{n}\right) \in \mathbb{R}^{n}-\{0\} .
\end{aligned}
$$

In the isotropic case (i.e. $a_{i p j q}=\mu\left(\delta_{p q} \delta_{i j}+\delta_{i q} \delta_{j p}\right)+\lambda \delta_{i p} \delta_{j q}, \lambda+\frac{2}{n} \mu>0$ and $\mu>0$ ), the above assumptions are all satisfied. Let $\left\{\lambda_{l}(\xi)\right\}_{l=1, \ldots, d}$ be the distinct eigenvalues of $\sum_{i, j=1}^{n} a_{i j} \xi_{i} \xi_{j}$. Then, $\lambda_{l}(\xi)$ become positive $C^{\infty}$ functions $(\xi \neq 0)$. We denote by $P_{l}(\xi)$ the projection into the eigenspace of $\lambda_{l}(\xi)$. 
For each $\lambda_{l}$ there are waves of $\lambda_{l}$-mode with the corresponding propagation speed. One of the main purposes in this paper is to examine how the waves are reflected near the boundary when the plane wave of a single mode hits the boundary perpendicularly. In Sect. 2 we make this investigation in terms of asymptotic solutions of the form

$$
u^{l}(t, x ; \sigma)=\sum_{j=0}^{\infty} e^{i \sigma\left(\varphi^{l}(x)-t\right)} u_{j}^{l}(t, x)(i \sigma)^{-j},
$$

where $\varphi^{l}(x)$ is a $C^{\infty}$ real-valued function satisfying $\lambda_{l}\left(\partial_{x} \varphi^{l}(x)\right)=1$. In this paper "the wave of $\lambda_{l}$-mode" means the asymptotic solution of this type. The typical plane wave of $\lambda_{k}$-mode is expressed of the form

$$
v(t, x ; \sigma)=e^{i \sigma\left(\lambda_{k}(\omega)^{-1 / 2} \omega \cdot x-t\right)} v_{0}
$$

with $\omega \in S^{n-1}$ and $v_{0}=P_{k}(\omega) v_{0}$, which propagates in the direction $\omega$ with the speed $\lambda_{l}(\omega)^{1 / 2}$.

We fix the above $k, \omega$, and $v_{0}(\neq 0)$, and construct the solutions $u^{l}(t, x ; \sigma)$ $(l=1, \ldots, d)$ so that $\sum_{l=1}^{d} u^{l}(t, x ; \sigma)+v(t, x ; \sigma)$ satisfies the boundary condition. Then, generally, every part $u^{l}$ does not vanish, that is, the mode-conversion happens. However, in special situations, this conversion does not occur; e.g., when $\Omega$ is the half-space and $\omega$ is normal to the boundary, all $u^{l}$ except $u^{k}$ vanish. This suggests that the waves $u^{l}$ in general cases with $l \neq k$ vanish on the characteristic curves starting at points on $\partial \Omega$ where the incident wave $v$ hits $\partial \Omega$ perpendicularly. This is correct if $\partial \Omega$ is flat there (cf. Theorem 2.1 and the corollary). But, if $\partial \Omega$ is not flat at those points, this conjecture is not correct (cf. Remark 2.2). We note that the asymptotic solutions are unique (see Theorem 1.2).

Section 1 is devoted to construction of the asymptotic solutions with nonglancing given data on $\partial \Omega$. We carry out this construction for more general operators with the variable coefficients. In the isotropic case, Karal and Keller [2] made such solutions. It seems difficult to apply their methods to our case. The essence of our methods is similar to Lax's [3]. However, the transport equations in our case are of different type, that is, they become first order equations with matrix-valued (not scalar-valued) coefficients. To solve these equations, we introduce the local coordinates associated with the bicharacteristic curves, and reduce them to symmetric differential equations ordinary in the space variables.

The asymptotic solutions in Sect. 1 seem useful for studies of various problems. By a similar idea to ours, Soga [5] has constructed the asymptotic solutions under more restricted assumptions, and used them to solve an inverse scattering problem. But the analysis in [5] is not so precise as to obtain the results in the present paper.

\section{Construction of the Asymptotic Solutions}

We set $\mathscr{B}^{\infty}(M)=\left\{f(x) \in C^{\infty}(M) ; \sup _{x \in M}\left|\partial_{x}^{\alpha} f(x)\right|<\infty\right.$ for any $\left.\alpha\right\}$. Let $a_{i j}(x), b_{i}(x)$ and $c(x)(i, j=1, \ldots, n)$ be $n \times n$-matrices of functions $\in \mathscr{B}^{\infty}\left(\mathbb{R}^{n}\right)$, and put

$$
L\left(x, \partial_{x}\right) u=\sum_{i, j=1}^{n} a_{i j}(x) \partial_{x_{i}} \partial_{x_{j}} u+\sum_{i=1}^{n} b_{i}(x) \partial_{x_{i}} u+c(x) u .
$$


We assume that the $(p, q)$-components $a_{i p j q}(x)$ of $a_{i j}(x)$ are real-valued and for each $x \in \mathbb{R}^{n}$ satisfy the conditions (A.1) (A.3) in introduction. In view of (A.3), we denote by $\lambda_{l}(x, \xi)(l=1, \ldots, d)$ the distinct eigenvalues of $\sum_{i, j=1}^{n} a_{i j}(x) \xi_{i} \xi_{j}\left(\lambda_{1}<\cdots<\lambda_{d}\right)$. By (A.1) $\sim\left(\right.$ A.3), $\lambda_{l}(x, \xi)$ become positive $C^{\infty}$ functions homogeneous of order 2 in $\xi$. Let $P_{l}(x, \xi)$ be the orthogonal projection into the eigenspace of $\lambda_{l}(x, \xi)$, which is an $n \times n$-matrix of $C^{\infty}$ functions homogeneous of order 0 in $\xi$. $\lambda_{l}(x, \xi)$ and $P_{l}(x, \xi)$ are even in $\xi$ (i.e. $\lambda_{l}(x, \xi)=\lambda_{l}(x,-\xi)$ and $P_{l}(x, \xi)=P_{l}(x,-\xi)$ ).

Let $U$ be an open set in $\mathbb{R}^{n}(U \cap \partial \Omega \neq \phi)$, and consider the following equation for each $\lambda_{l}(x, \xi)$ :

$$
\left\{\begin{array}{llr}
\lambda_{l}\left(x, \partial_{x} \varphi^{l}(x)\right)=1 & \text { in } & \Omega \cap U, \\
\varphi^{l}\left(x^{\prime}\right)=\varphi\left(x^{\prime}\right) & \text { on } & \partial \Omega \cap U, \\
\frac{\partial \varphi^{l}}{\partial v}\left(x^{\prime}\right)<0 & \text { on } & \partial \Omega \cap U,
\end{array}\right.
$$

where $\varphi\left(x^{\prime}\right)$ is a given $C^{\infty}$ real-valued function and $v\left(x^{\prime}\right)={ }^{t}\left(v_{1}\left(x^{\prime}\right), \ldots, v_{n}\left(x^{\prime}\right)\right)$ is the unit outer vector normal to $\partial \Omega$ at $x^{\prime} \in \partial \Omega$ (the points on $\partial \Omega$ being denoted by $\left.x^{\prime}=\left(x_{1}^{\prime}, \ldots, x_{n}^{\prime}\right)\right)$. As is well known, if the (first order) derivatives of $\varphi\left(x^{\prime}\right)$ along the boundary are near 0 on $\partial \Omega \cap U$, we can construct a (unique) real-valued solution $\varphi^{l}(x)$ of (1.1) in a neighborhood of the boundary by the Hamilton-Jacobi methods; we assume that the $U$ is chosen so that $\varphi^{l}(x)$ is defined on whole $\Omega \cap U$. There exists an implicit function $\tau_{l}\left(x^{\prime}, \xi\right)$ defined on $(\partial \Omega \cap U) \times \Lambda$ (where $\Lambda\left(\subset \mathbb{R}^{n}\right)$ is a small neighborhood of $\xi=0$ ) such that

$$
\lambda_{l}\left(x^{\prime}, \tau_{l}\left(x^{\prime}, \xi\right) v\left(x^{\prime}\right)+\xi\right)=1 \quad \text { and } \quad \tau_{l}\left(x^{\prime}, \xi\right)<0 .
$$

Let us note that for $x^{\prime} \in \partial \Omega \cap U$,

$$
\begin{aligned}
\tau_{l}\left(x^{\prime}, 0\right) & =-\lambda_{l}\left(x^{\prime}, v\left(x^{\prime}\right)\right)^{-1 / 2}, \\
\partial_{x} \varphi^{l}\left(x^{\prime}\right) & =\tau_{l}\left(x^{\prime}, \partial_{x} \varphi_{\tan }^{l}\left(x^{\prime}\right)\right) v\left(x^{\prime}\right)+\partial_{x} \varphi_{\tan }^{l}\left(x^{\prime}\right),
\end{aligned}
$$

where $\partial_{x} \varphi_{\tan }^{l}\left(x^{\prime}\right)$ denotes the tangential part (to $\partial \Omega$ ) of $\partial_{x} \varphi^{l}\left(x^{\prime}\right)$.

We impose the boundary condition $B u=g$ on $\partial \Omega \cap U$, where

$$
\begin{aligned}
B u & =\left.u\right|_{\partial \Omega} \text { or }=\left.(N+b) u\right|_{\partial \Omega}, \\
N & =\sum_{i, j=1}^{n} a_{i j} v_{i} \partial_{x_{j}}, \quad b=b(x) \in \mathscr{B}^{\infty}(\partial \Omega) .
\end{aligned}
$$

If a function $f(x ; \sigma) \in \mathscr{B}^{0}(M)$ with a parameter $\sigma \in \mathbb{R}$ satisfies $|f(\cdot, \sigma)|_{\mathscr{B}^{\circ}(M)} \leqq C|\sigma|^{m}$ for $|\sigma| \geqq 1$, we write ' $f(x ; \sigma)=O\left(|\sigma|^{m}\right)$ in $M^{\prime}$ '. 'The asymptotic solution $u^{l}(t, x ; \sigma)=$ $\sum_{j=0}^{\infty} e^{i \sigma\left(\varphi^{l}(x)-t\right)} u_{j}^{l}(t, x)(i \sigma)^{-j}$ (in $\mathbb{R} \times(U \cap \Omega)$ and of $\lambda_{l}$-mode) with the boundary data $g\left(t, x^{\prime} ; \sigma\right)=\sum_{j=0}^{\infty} e^{i \sigma\left(\varphi\left(x^{\prime}\right)-t\right)} g_{j}\left(t, x^{\prime}\right)(i \sigma)^{-j+\varepsilon^{\prime}}$ means that

$$
\begin{cases}\left(\partial_{t}^{2}-L\right)\left(\sum_{j=0}^{m} e^{i \sigma\left(\varphi^{l}-t\right)} u_{j}^{l}(i \sigma)^{-j}\right)=O\left(|\sigma|^{-m+m_{0}}\right) & \text { in } \quad \mathbb{R} \times(\Omega \cap U), \\
B\left\{\sum_{j=0}^{m} e^{i \sigma\left(\varphi^{l}-t\right)} u_{j}^{l}(i \sigma)^{-j}\right\}-\left\{\sum_{j=0}^{m} e^{i \sigma\left(\varphi\left(x^{\prime}\right)-t\right)} g_{j}\left(t, x^{\prime}\right)(i \sigma)^{-j+\varepsilon}\right\}=O\left(|\sigma|^{-m+m_{1}}\right) & \begin{array}{l}
\text { on } \\
\mathbb{R} \times(\partial \Omega \cap U)
\end{array}\end{cases}
$$


for any positive integer $m$, where $\varepsilon=0$ (respectively $=1$ ) when $B u=\left.u\right|_{\partial \Omega}$ (respectively when $\left.B u=\left.(N+b) u\right|_{\partial \Omega}\right)$, and $m_{0}, m_{1}$ are constants independent of $m$. And we write

$$
\left\{\begin{array}{lll}
\left(\partial_{t}^{2}-L\right) u^{l}(t, x ; \sigma) \cong 0 & \text { in } \quad \mathbb{R} \times(\Omega \cap U), \\
B u^{l}\left(t, x^{\prime} ; \sigma\right) \cong g\left(t, x^{\prime} ; \sigma\right) & \text { on } \quad \mathbb{R} \times(\partial \Omega \cap U) .
\end{array}\right.
$$

Theorem 1.1. Assume that the (first order) derivatives of $\varphi\left(x^{\prime}\right)$ (in (1.1)) along the boundary are near 0 on $\partial \Omega \cap U$. Then, for any data $g\left(t, x^{\prime} ; \sigma\right)=\sum_{j=0}^{\infty} e^{i \sigma\left(\varphi\left(x^{\prime}\right)-t\right)}$ $g_{j}\left(t, x^{\prime}\right)(i \sigma)^{-j+\varepsilon}\left(g_{j}\left(t, x^{\prime}\right) \in \mathscr{B}{ }^{\infty}(\mathbb{R} \times(\partial \Omega \cap U)) ; \varepsilon=0\right.$ (respectively=1) when $B u=\left.u\right|_{\partial \Omega}$ (respectively when $\left.B u=\left.(N+b) u\right|_{\partial \Omega}\right)$ ), we have asymptotic solutions $u^{l}(t, x ; \sigma)=$ $\sum_{j=0}^{\infty} e^{i \sigma\left(\varphi^{l}(x)-t\right)} u_{j}^{l}(t, x)(i \sigma)^{-j}(l=1, \ldots, d)$ such that

$$
\begin{array}{ll}
\left(\partial_{t}^{2}-L\right) u^{l}(t, x ; \sigma) \cong 0 & \text { in } \quad \mathbb{R} \times(\Omega \cap U), \\
\sum_{l=1}^{d} B u^{l}\left(t, x^{\prime} ; \sigma\right) \cong g\left(t, x^{\prime} ; \sigma\right) & \text { on } \quad \mathbb{R} \times(\partial \Omega \cap U),
\end{array}
$$

(iii) if $g\left(t, x^{\prime} ; \sigma\right)=0$ for $t<t_{0}$, then $u^{l}(t, x ; \sigma)=0$ for $t<t_{0}(l=1, \ldots, d)$.

Theorem 1.2. The asymptotic solutions $u_{d}^{l}(t, x ; \sigma)=\sum_{j=0}^{\infty} e^{i \sigma\left(\varphi^{l}-t\right)} u_{j}^{l}(i \sigma)^{-j}(l=1, \ldots, d)$ in Theorem 1.1 are unique: If $\left(\partial_{t}^{2}-L\right) \sum_{l=1}^{d} u^{l} \cong 0$ in $\mathbb{R}_{j=0} \times(\Omega \cap U)$ and $B \sum_{l=1}^{d} u^{l} \cong 0$ on $\mathbb{R} \times(\partial \Omega \cap U)$, then $u_{j}^{l}(t, x)=0$ in $\mathbb{R} \times(\Omega \cap U)$ for $l=1, \ldots, d$ and $j=0,1, \ldots$

Theorem 1.2 will be verified after proving Theorem 1.1.

Let us prove Theorem 1.1. At first, note that the solution $\varphi^{l}(x)$ of (1.1) exists for every $l(=1, \ldots, d)$ since the derivatives of $\varphi\left(x^{\prime}\right)$ are near 0 .

Hereafter we use the notations $f_{x}\left(=\partial_{x} f\right)$ and $f_{x_{i}}\left(=\partial_{x_{i}} f\right)$. We rewrite $\left(\partial_{t}^{2}-L\right) \sum_{j=0}^{\infty} e^{i \sigma\left(\varphi^{l}-t\right)} u_{j}^{l}(i \sigma)^{-j}$ in the following form:

$$
\begin{aligned}
& e^{i \sigma\left(\varphi^{l}(x)-t\right)} \sum_{j=0}^{\infty}\left[\left(I-\sum a_{p q} \varphi_{x_{p}}^{l} \varphi_{x_{q}}^{l}\right) P_{l} u_{j}^{l}+\left(I-\sum a_{p q} \varphi_{x_{p}}^{l} \varphi_{x_{q}}^{l}\right)\left(I-P_{l}\right) u_{j}^{l}\right. \\
& \quad+\left(I-P_{l}\right)\left\{\left(-2 \partial_{t}-H\right) u_{j-1}^{l}+\left(\partial_{t}^{2}-L\right) u_{j-2}^{l}\right\}+P_{l}\left(-2 \partial_{t}-H\right) P_{l} u_{j-1}^{l} \\
& \left.\quad+P_{l}\left\{\left(-2 \partial_{t}-H\right)\left(I-P_{l}\right) u_{j-1}^{l}+\left(\partial_{t}^{2}-L\right) u_{j-2}^{l}\right\}\right](i \sigma)^{-j+2}
\end{aligned}
$$

where $u_{-1}=u_{-2}=0, P_{l}=P_{l}\left(x, \varphi_{x}^{l}(x)\right)$ and

$$
H=\sum_{i, j=1}^{n}\left(a_{i j}(x)+a_{j i}(x)\right) \varphi_{x_{i}}^{l}(x) \partial_{x_{j}}+(L-c) \varphi^{l} .
$$

In the same way as Lax [3], we determine $\left(I-P_{l}\right) u_{j}^{l}$ and next $P_{l} u_{j}^{l}$, and eliminate each term in the above summation together with making $u_{j}^{l}$ satisfy the boundary condition. When considering the boundary condition, we use the matrices

$$
\begin{aligned}
& P\left(x^{\prime}\right)=\sum_{l=1}^{d} P_{l}\left(x^{\prime}, \varphi_{x}^{l}\left(x^{\prime}\right)\right), \\
& Q\left(x^{\prime}\right)=\sum_{l=1}^{d}\left(N \varphi^{l}\right)\left(x^{\prime}\right) P_{l}\left(x^{\prime}, \varphi_{x}^{l}\left(x^{\prime}\right)\right) .
\end{aligned}
$$


Since $P\left(x^{\prime}\right)=\sum_{l=1}^{d} P_{l}\left(x^{\prime}, v\left(x^{\prime}\right)\right)$ and $Q\left(x^{\prime}\right)=-\sum_{l=1}^{d} \lambda_{l}\left(v\left(x^{\prime}\right)\right)^{1 / 2} P_{l}\left(x^{\prime}, v\left(x^{\prime}\right)\right)$ if the derivatives of $\varphi\left(x^{\prime}\right)$ all vanish, $P\left(x^{\prime}\right)$ and $Q\left(x^{\prime}\right)$ are non-singular when its derivatives are near 0 on $\partial \Omega \cap U$.

It is obvious that the part $\left(I-\sum a_{p q} \varphi_{x_{p}}^{l} \varphi_{x_{q}}^{l}\right) P_{l} u_{j}^{l}$ is equal to $\left(I-\lambda_{l} I\right) P_{l} u_{j}^{l}=0$. To eliminate the other parts, we consider the equations

$$
P_{l} u_{j}^{l}=\left\{\begin{array}{r}
P_{l}\left(2 \partial_{t}+H\right) P_{l} u_{j}^{l}+P_{l} f_{j}^{l}=0 \text { in } \mathbb{R} \times(\Omega \cap U), \\
P_{l} P^{-1}\left(-\sum_{i=1}^{d}\left(I-P_{i}\right) u_{j}^{i}+g_{j}\right) \text { on } \mathbb{R} \times(\partial \Omega \cap U) \quad \text { when } B u=\left.u\right|_{\partial \Omega}, \\
P_{l} Q^{-1}\left(-\sum_{i=1}^{d}\left(N \varphi^{i}\right)\left(I-P_{i}\right) u_{j}^{i}-\sum_{i=1}^{d} B u_{j-1}^{i}+g_{j}\right) \\
\text { on } \mathbb{R} \times(\partial \Omega \cap U) \text { when } B u=\left.(N+b) u\right|_{\partial \Omega},
\end{array}\right.
$$

$$
\left(I-\sum a_{p q} \varphi_{x_{p}}^{l} \varphi_{x_{q}}^{l}\right)\left(I-P_{l}\right) u_{j}^{l}+\left(I-P_{l}\right) h_{j-1}^{l}=0 \quad \text { in } \quad \mathbb{R} \times(\Omega \cap U),
$$

where $f_{j}^{l}=\left(2 \partial_{t}+H\right)\left(I-P_{l}\right) u_{j}^{l}-\left(\partial_{t}^{2}-L\right) u_{j-1}^{l}$ and $h_{j-1}^{l}=\left(-2 \partial_{t}-H\right) u_{j-1}^{l}+\left(\partial_{t}^{2}-L\right) u_{j-2}^{l}$. Note that $f_{j}^{l}$ and $h_{j-1}^{l}$ are defined from only $u_{j-2}^{l}, u_{j-1}^{l}$ and $\left(I-P_{l}\right) u_{j}^{l}$. At first put $\left(I-P_{l}\right) u_{0}^{l}=0$; i.e. take $u_{0}^{l}$ in the eigenspace $P_{l} \mathbb{R}^{n}$. Then $f_{0}^{l}=0$. If Eqs. (1.3) and (1.4) for $j=0$ are solvable, $u_{0}^{l}$ is determined. Noting that $\left(I-P_{l}\right)=\sum_{i \neq l} P_{i}$, we see that $(1.5)$ is of the form $\sum_{l \neq l}\left\{\left(1-\lambda_{i}\right) P_{i} u_{1}^{l}+P_{i} h_{0}^{l}\right\}$. Therefore, setting $P_{i} u_{1}^{l}=$ $-\left(1-\lambda_{i}\right)^{-1} P_{i} h_{0}^{l}$ (for $\left.i \neq l\right)$ determines $\left(I-P_{l}\right) u_{1}^{l}$. Repeating this procedure inductively, we can determine all $u_{j}^{l}(j=0,1, \ldots)$. Furthermore it is seen that the asymptotic solution $\sum_{l=1}^{d} u^{l}(t, x ; \sigma)$ with these $u_{j}^{l}(t, x)$ satisfy the required boundary conditions: In fact, when $B u=\left.u\right|_{\partial \Omega},(1.4)$ yields that

$$
\begin{aligned}
\sum_{l=1}^{d} \sum_{j=0}^{m} e^{i \sigma\left(\varphi^{l}-t\right)} u_{j}^{l}(i \sigma)^{-j} & =e^{i \sigma(\varphi-t)} \sum_{j=0}^{m} \sum_{l=1}^{d}\left\{P_{l} u_{j}^{l}+\sum_{l=1}^{d}\left(I-P_{l}\right) u_{j}^{l}\right\}(i \sigma)^{-j} \\
& =\sum_{j=0}^{m} e^{i \sigma(\varphi-t)} g_{j}(i \sigma)^{-j} \text { on } \mathbb{R} \times(\partial \Omega \cap U)
\end{aligned}
$$

when $B u=\left.(N+b) u\right|_{\partial \Omega}$,

$$
\begin{aligned}
& B \sum_{l=1}^{d} \sum_{j=0}^{m} e^{i \sigma\left(\varphi^{l}-t\right)} u_{j}^{l}(i \sigma)^{-j}=e^{i \sigma(\varphi-t)} \sum_{j=0}^{m}\left\{\sum_{l=1}^{d}\left(N \varphi^{l}\right) P_{l} u_{j}^{l}+\right. \\
& \left.\sum_{l=1}^{d}\left(N \varphi^{l}\right)\left(I-P_{l}\right) u_{j}^{l}+\sum_{l=1}^{d} B u_{j-1}^{l}\right\}(i \sigma)^{-j+1}+e^{i \sigma(\varphi-t)} \sum_{l=1}^{d} B u_{m}^{l}(i \sigma)^{-m} \\
& =\sum_{j=0}^{m} e^{i \sigma(\varphi-t)} g_{j}(i \sigma)^{-j+1}+e^{i \sigma(\varphi-t)} \sum_{l=1}^{d} B u_{m}^{l}(i \sigma)^{-m} \text { on } \mathbb{R} \times(\partial \Omega \cap U) .
\end{aligned}
$$

Hence we have only to show that (1.3) and (1.4) are solvable for each $l=1, \ldots, d$. Fix $\tilde{x} \in \partial \Omega \cap U$ and take bases $\tilde{e}_{i}(i=1, \ldots, \tilde{d})$ in $P_{l}(\tilde{x} ; v(\tilde{x})) \mathbb{R}^{n}$. Then 
$e_{i}^{\prime}(x)=P_{l}\left(x, \varphi_{x}^{l}(x)\right) \tilde{e}_{i}(i=1, \ldots, \tilde{d})$ are linearly independent if the (first order) derivatives of $\varphi\left(x^{\prime}\right)$ are near 0 and $x$ moves near $\tilde{x}$. We assume that $e_{i}^{\prime}(x)$ are linearly independent for $x \in \Omega \cap U$. Setting

$$
e_{1}=\left|e_{1}^{\prime}\right|^{-1} e_{1}^{\prime}, e_{i}=\left|e_{i}^{\prime}-\sum_{j=1}^{i-1}\left({ }^{t} e_{i}^{\prime} e_{j}\right) e_{j}\right|^{-1}\left(e_{i}^{\prime}-\sum_{j=1}^{i-1}\left({ }^{t} e_{i}^{\prime} e_{j}\right) e_{j}\right) \quad(i=2, \ldots, \tilde{d}),
$$

we obtain orthonormal bases $e_{i}(x)(i=1, \ldots, \widetilde{d})$ in $P_{l}\left(x, \varphi_{x}^{l}(x)\right) \mathbb{R}^{n}$, which belong to $\mathscr{B}^{\infty}(\Omega \cap U)$. We put

$$
\begin{aligned}
e(x) & =\left(e_{1}(x), \ldots, e_{\tilde{d}}(x)\right), \\
v_{j}(t, x) & ={ }^{t} e(x) u_{j}^{l}(t, x) \quad(j=0,1, \ldots) .
\end{aligned}
$$

Let $(q(s ; y), p(s ; y))$ be the bicharacteristic curves defined by

$$
\begin{cases}\frac{d q}{d s}=\partial_{\xi} \lambda_{l}(q, p), & \frac{d p}{d s}=-\partial_{x} \lambda_{l}(q, p) \\ q(0 ; y)=x^{\prime}(y), & p(0 ; y)=\varphi_{x}^{l}\left(x^{\prime}(y)\right),\end{cases}
$$

where $x^{\prime}(y)$ is a system of local coordinates of $\partial \Omega \cap U\left(y \in \mathbb{R}^{n-1}\right)$. Then the mapping: $(s, y) \rightarrow q(s ; y)$ becomes diffeomorphic if the (first order) derivatives of $\varphi\left(x^{\prime}\right)$ are near 0 and $|s|$ are small enough. We assume that $x=q(s ; y)$ becomes a system of the local coordinates of $\Omega \cap U$. By using the variable $(s, y)$ and $w(s, t, y)=v_{j}(t, q(s ; y))$, Eqs. (1.3) and (1.4) are transformed as follows:

$$
\left\{2 \partial_{t}+a_{0}(s, y) \partial_{s}+\sum_{i=1}^{n-1} a_{i}(s, y) \partial_{y_{i}}+b_{0}(s, y)\right\} w(s, t, y)=f(s, t, y) \quad \text { in } \quad\left(0, s_{0}\right) \times \mathbb{R} \times \tilde{U}
$$

$$
w(0, t, y)=h(t, y) \quad \text { on } \quad \mathbb{R} \times \tilde{U},
$$

where $\Omega \cap U \subset\left\{q(s ; y):(s, y) \in\left(0, s_{0}\right) \times \tilde{U}\right\}$ and

$$
\begin{aligned}
& a_{0}={ }^{t} e \sum_{i, j=1}^{n}\left(a_{i j}+a_{j i}\right) \varphi_{x_{i}}^{l} \frac{\partial s}{\partial x_{j}} e \\
& a_{i}={ }^{t} e \sum_{p, j=1}^{n}\left(a_{p j}+a_{j p}\right) \varphi_{x_{p}}^{l} \frac{\partial y_{i}}{\partial x_{j}} e \quad(i=1, \ldots, n-1), \\
& b_{0}={ }^{t} e\left(L \varphi^{l}-c \varphi^{l}\right) e+{ }^{t} e \sum_{i, j=1}^{n}\left(a_{i j}+a_{j i}\right) \varphi_{x_{i}}^{l} \frac{\partial s}{\partial x_{i}} \partial_{s} e \\
& \quad+{ }^{t} e \sum_{i=1}^{n-1} \sum_{p, j=1}^{n}\left(a_{p j}+a_{j p}\right) \varphi_{x_{p}}^{l} \frac{\partial y_{i}}{\partial x_{j}} \partial_{y_{i}} e .
\end{aligned}
$$

Solving this equation with $f=-{ }^{t} e f_{j}^{l}$ and $h={ }^{t} e P^{-1}\left(-\sum_{i=1}^{d}\left(I-P_{i}\right) u_{j}^{i}+g_{j}\right)$ (respectively $={ }^{t} e Q^{-1}\left(-\sum_{i=1}^{d}\left(N \varphi^{i}\right)\left(I-P_{i}\right) u_{j}^{i}-\sum_{i=1}^{d} B u_{j-1}^{i}+g_{j}\right)$ when $B u=\left.u\right|_{\partial \Omega}$ (respectively when $\left.B u=\left.(N+b) u\right|_{\partial \Omega}\right)$, we get the required solution $P_{l} u_{j}^{l}=e w$ for each $l(=1, \ldots, d)$.

Now, let us examine (1.8). At first, note that every $a_{i}(i=1, \ldots, n-1)$ defined 
by (1.10) vanishes: $(1.8)$ is reduced to

$$
\left(2 \partial_{t}+a_{0} \partial_{s}+b_{0}\right) w=f \text { in }\left(0, s_{0}\right) \times \mathbb{R} \times \tilde{U},
$$

which is seen from

Lemma 1.3. If $\xi$ and $\eta \in \mathbb{R}^{n}(\xi \neq 0)$ satisfy $\eta \cdot \partial_{\xi} \lambda_{l}(x, \xi)=0$ ( $x$ and $l$ being fixed arbitrarily), then we have

$$
P_{l}(x, \xi) \sum_{i, j=1}^{n}\left(a_{i j}(x)+a_{j i}(x)\right) \xi_{i} \eta_{j} P_{l}(x, \xi)=0 .
$$

For the local coordinates $x=q(s ; y)$ we have $\left(\partial_{x} y_{i}\right) \cdot\left(\partial_{\xi} \lambda_{l}\left(x, \varphi_{x}^{l}\right)\right)=\left(\partial_{x} y_{i}\right) \cdot\left(\partial_{s} q\right)=0$ $(i=1, \ldots, n-1)$; using the above lemma with $\xi=\varphi_{x}^{l}$ and $\eta=\partial_{x} y_{i}$, we obtain $P_{l}\left(x, \varphi_{x}^{l}\right) \sum_{k, j=1}^{n}\left(a_{k j}+a_{j k}\right) \varphi_{x_{k}}^{l} \frac{\partial y_{i}}{\partial x_{j}} P_{l}\left(x, \varphi_{x}^{l}\right)=0$, which yields that $a_{i}=0(i=1, \ldots, n-1)$.

Proof of Lemma 1.3. Applying $\partial_{\xi_{p}}$ to the equality

$$
\sum_{i, j=1}^{n} a_{i j}(x) \xi_{i} \xi_{j} P_{l}(x, \xi)=\lambda_{l}(x, \xi) P_{l}(x, \xi)
$$

we obtain the equality $\sum_{i=1}^{n}\left(a_{i p}+a_{p i}\right) \xi_{i} P_{l}+\sum_{i, j=1}^{n} a_{i j}(x) \xi_{i} \xi_{j} \partial_{\xi_{p}} P_{l}=\left(\partial_{\xi_{p}} \lambda_{l}\right) P_{l}+\lambda_{l} \partial_{\xi_{p}} P_{l}$. Multiply this equality by $\eta_{p}$ and sum it up in $p=1, \ldots, n$. Then we have

$$
\begin{aligned}
P_{l} & \sum_{i, p=1}^{n}\left(a_{i p}+a_{p i}\right) \xi_{i} \eta_{p} P_{l} \\
\quad= & \left(\sum_{p=1}^{n} \eta_{p} \partial_{\xi_{p}} \lambda_{l}\right) P_{l}+\lambda_{l} P_{l} \sum_{p=1}^{n} \eta_{p} \partial_{\xi_{p}} P_{l}-P_{l} \sum_{i, j=1}^{n} a_{i j}(x) \xi_{i} \xi_{j} \sum_{p=1}^{n} \eta_{p} \partial_{\xi_{p}} P_{l}=0 .
\end{aligned}
$$

The proof is complete.

Next, let us note that $a_{0}(s, y)$ is of the special form:

Lemma 1.4. $a_{0}(s, y)$ (defined by (1.9)) is a symmetric $\tilde{d} \times \tilde{d}$-matrix of real-valued functions, and satisfies

$$
\left|a_{0}(s, y)-I\right|_{\mathscr{B}^{0}\left(\left(0, s_{0}\right) \times \tilde{U}\right)} \leqq C\left|\partial_{y} \varphi\left(x^{\prime}(y)\right)\right|_{\mathscr{B}^{0}(\tilde{U})} .
$$

Proof. Let $(q(s ; y), p(s ; y))$ be the solution of $(1.7)$. Then, $p(s ; y)=\varphi_{x}^{l}(q(s ; y))$ and $\lambda_{l}(q(s ; y), p(s ; y))=1$. By the Euler equality, we have $2=\partial_{\xi} \lambda \cdot p=\partial_{s} q \cdot p$. Differentiating this in $y_{i}$ yields that $\partial_{s} \partial_{y_{i}} q \cdot p+\partial_{s} q \cdot \partial_{y_{i}} p=0$. In the same way, we obtain $\partial_{y_{i}} q \cdot \partial_{s} p-\partial_{s} q \cdot \partial_{y_{i}} p=0$ from the equality $\lambda_{l}(q, p)=1$. Therefore, $\partial_{s}\left(\partial_{y_{i}} q \cdot p\right)=0$. Combining this euality with (1.2), we have

$$
\left|\partial_{y_{i}} q \cdot p\right| \leqq C_{1}\left|\partial_{y} \varphi\left(x^{\prime}(y)\right)\right|_{\mathscr{B}^{0}(\tilde{U})}
$$

Let $\mu(s, y)$ be the unit vector orthogonal to every $\partial_{y_{i}} q(s ; y)(i=1, \ldots, n-1)$ with $v \cdot \mu>0 . \gamma={ }^{t}\left(\frac{\partial s}{\partial x_{1}}, \ldots, \frac{\partial s}{\partial x_{n}}\right)$ satisfies $\gamma \cdot \partial_{y_{i}} q=0(x=q(s ; y))$, which implies that $\gamma=-|\gamma| \mu$. From this and the equality $p=(p \cdot \mu) \mu+(p-(p \cdot \mu) \mu)$, we have

$$
\begin{aligned}
\gamma & =-|\gamma|(p \cdot \mu)^{-1} p+|\gamma|(p \cdot \mu)^{-1}(p-(p \cdot \mu) \mu) \\
& \equiv-|\gamma|(p \cdot \mu)^{-1} p+\tilde{\gamma} .
\end{aligned}
$$


Since $\tilde{\gamma}$ is expressed by linear combination of $\partial_{y_{1}} q, \ldots, \partial_{y_{n-1}} q$ and satisfies $\tilde{\gamma} \cdot \partial_{y_{i}} q=|\gamma|(p \cdot \mu)^{-1} \partial_{y_{i}} q \cdot p,(1.12)$ yields that

$$
|\tilde{\gamma}| \leqq C_{2}\left|\partial_{y} \varphi\left(x^{\prime}(y)\right)\right|_{\mathscr{B}^{0}(\tilde{U})} .
$$

Insert (1.13) into $1=\gamma \cdot \partial_{s} q$. Then $1=-|\gamma|(p \cdot \mu)^{-1} p \cdot \partial_{s} q+\tilde{\gamma} \cdot \partial_{s} q=-2|\gamma|(p \cdot \mu)^{-1}+$ $\tilde{\gamma} \cdot \partial_{s} q$. Therefore, using (1.13) again, we have

$$
\gamma=2^{-1} p-2^{-1}\left(\tilde{\gamma} \cdot \partial_{s} q\right) p+\tilde{\gamma}
$$

Mixing this with (1.14), we get

$$
\left|\frac{\partial s}{\partial x_{i}}-\frac{1}{2} \varphi_{x_{i}}^{l}\right| \leqq C_{3}\left|\partial_{y} \varphi\left(x^{\prime}(y)\right)\right|_{\mathscr{B}^{0}(\tilde{U})},
$$

which proves the lemma. The proof is complete.

Thus it suffices to analyze the following problem:

$$
\begin{cases}\left(2 \partial_{t}+\alpha(s) \partial_{s}+\beta(s)\right) w(s, t)=f(s, t) & \text { in } I \times \mathbb{R} \quad\left(I=\left(0, s_{0}\right)\right), \\ w(0, t)=h(t) & \text { on } \mathbb{R},\end{cases}
$$

where $\alpha$ and $\beta$ are $\tilde{d} \times \tilde{d}$-matrices of functions in $\mathscr{B}^{\infty}(I)$ and $\alpha$ is real-valued and symmetric. As is easily seen, all the requirements are derived from

Lemma 1.5. (i) If $|\operatorname{det} \alpha| \geqq \alpha_{0}(>0)$, there exists a unique solution $w(s, t)$ of $(1.15)$ in $\mathscr{B}^{\infty}(I \times \mathbb{R})$ for any $f(s, t) \in \mathscr{B}^{\infty}(I \times \mathbb{R})$ and $h(t) \in \mathscr{B}^{\infty}(\mathbb{R})$.

(ii) Let $2^{-1}|\zeta|^{2} \leqq t \zeta \alpha \zeta$ for $\zeta \in \mathbb{R}^{\tilde{d}}$. Then, if the support of the data $(f, h)$ in $(1.15)$ is contained in $\left\{t_{0} \leqq t\right\}$, so is the support of the solution $w$ of (1.15).

Proof. (i) From the assumption, $\alpha^{-1}(s)$ becomes a symmetric matrix of functions $\in \mathscr{B}^{\infty}(I)$. Equation (1.15) is equivalent to

$$
\begin{cases}\left(\partial_{s}+2 \alpha^{-1} \partial_{t}+\alpha^{-1} \beta\right) w=f & \text { in } I \times \mathbb{R}, \\ \left.w\right|_{s=0}=h & \text { on } \mathbb{R} .\end{cases}
$$

This is a well-known Cauchy problem (cf. Friedrichs [1] or Chap. 5 of Mizohata [4]). Therefore (i) in the lemma is obtained.

(ii) Introduce the variables $s^{\prime}=s$ and $t^{\prime}=2^{-1} t-s$. Then (1.15) is transformed into the problem

$$
\begin{cases}\left(\partial_{s^{\prime}}+\left(\alpha^{-1}-I\right) \partial_{t^{\prime}}+\alpha^{-1} \beta\right) w^{\prime}\left(s^{\prime}, t^{\prime}\right)=f^{\prime}\left(s^{\prime}, t^{\prime}\right) & \text { in } I \times \mathbb{R}, \\ \left.w^{\prime}\right|_{s^{\prime}=0}=h\left(t^{\prime}\right) & \text { on } \mathbb{R} .\end{cases}
$$

It is seen from the assumption that $\alpha^{-1}-I$ is a symmetric matrix satisfying $\left|{ }^{t} \zeta\left(\alpha^{-1}-I\right) \zeta\right| \leqq|\zeta|^{2}$ for $\zeta \in \mathbb{R}^{\tilde{d}}$. This implies that every eigenvalue of this matrix is in $[-1,1]$. Therefore $(1.16)$ is not only solvable but also has finite propagation speed less than 1 (cf. Note 2 in Sect. 12 of Chap. 6 of [4]). Hence, in the original Eq. (1.15), the solution $w(s, t)$ exists and is equal to 0 on the cone $D=\{(s, t) \in I \times \mathbb{R}$; $\left.\left|2^{-1}\left(t-t_{1}\right)-s+s_{1}\right| \leqq s_{1}-s, s<s_{1}\right\}$ if the data vanish on $D$, which proves (ii) in the lemma.

Proof of Theorem 1.2. Inserting $u=\sum_{j=0}^{\infty} \sum_{l=1}^{d} e^{i \sigma\left(\varphi^{l}-t\right)} u_{j}^{l}(i \sigma)^{-j}$ into $\left(\partial_{t}^{2}-L\right) u \cong 0$ and 
$B u \cong 0$, we have

$$
\begin{gathered}
\sum_{l=1}^{d} e^{i \sigma \varphi^{l}}\left\{\left(I-\sum a_{p q} \varphi_{x_{p}}^{l} \varphi_{x_{q}}^{l}\right) u_{j}^{l}-\left(2 \partial_{t}+H\right) u_{j-1}^{l}+\left(\partial_{t}^{2}-L\right) u_{j-2}^{l}\right\} \\
=0 \text { in } \mathbf{R} \times(\Omega \cap U), \\
\sum_{l=1}^{d} u_{j}^{l}=0 \text { on } \mathbb{R} \times(\partial \Omega \cap U) \text { when } B u=\left.u\right|_{\partial \Omega}, \\
\sum_{l=1}^{d}\left\{\sum_{p, q=1}^{d} v_{p}\left(\varphi_{x_{q}}^{l} a_{p q} u_{j}^{l}+a_{p q} \partial_{x_{a}} u_{j-1}^{l}\right)+b u_{j-1}^{l}\right\}=0 \\
\text { on } \mathbb{R} \times(\partial \Omega \cap U) \text { when } B u=\left.(N+b) u\right|_{\partial \Omega},
\end{gathered}
$$

for any $j=0,1, \ldots$, and any large $\sigma$.

Take any pair $\varphi^{i}$ and $\varphi^{j}(1 \leqq i<j \leqq d)$. Then, for any open set $W \subset \Omega \cap U$ there exists a point $x$ in $W$ such that $\varphi^{i}(x) \neq \varphi^{j}(x)$; because, if not, $\varphi_{x}^{i}=\varphi_{x}^{j}$ in $W$ and so $1=\lambda_{i}\left(x, \varphi_{x}^{i}\right)=\lambda_{i}\left(x, \varphi_{x}^{j}\right)<\lambda_{j}\left(x, \varphi_{x}^{j}\right)=1$, which is a contradiction. This fact implies that the set $\widetilde{\Omega}=\left\{x: \varphi^{i}(x) \neq \varphi^{j}(x)\right.$ for every $i$ and $j$ with $\left.i \neq j\right\}$ is dense in $\Omega \cap U$. On the other hand, if $\varphi^{1}, \ldots, \varphi^{d}$ are different each other, each term (hereafter denoted by $\left.e^{i \sigma \varphi^{l}} \Phi^{l}\right)$ in the summation of (1.17) must be equal to 0 . In fact, differentiating $\sum_{l=1}^{d} e^{i \sigma \varphi^{l}} \Phi^{l}=0(d-1)$-times in $\sigma$, we have $\left(\Phi^{1}, \ldots, \Phi^{d}\right) J=0$, where $J$ is the $d \times d$ matrix whose $(l, k)$-component is $\left(i \varphi^{l}\right)^{k-1} e^{i \sigma \varphi^{l}}$. This yields that $\left(\Phi^{1}, \ldots, \Phi^{d}\right)=0$ since det $J \neq 0$. Therefore each term $e^{i \sigma \varphi^{l}} \Phi^{l}$ vanishes in $\mathbb{R} \times \widetilde{\Omega}$ and so in $\mathbb{R} \times(\Omega \cap U)$ (since $u_{j}$ are assumed smooth). Hence, rewriting the $\Phi^{l}$, we obtain from (1.17)

$$
\begin{aligned}
& \left(I-\sum a_{p q} \varphi_{x_{p}}^{l} \varphi_{x_{q}}^{l}\right)\left(I-P_{l}\right) u_{j}^{l}+\left(I-P_{l}\right)\left\{\left(-2 \partial_{t}-H\right) u_{j-1}^{l}+\left(\partial_{t}^{2}-L\right) u_{j-2}^{l}\right\} \\
& -P_{l}\left(2 \partial_{t}+H\right) P_{l} u_{j-1}^{l}+P_{l}\left\{\left(-2 \partial_{t}-H\right)\left(I-P_{l}\right) u_{j-1}^{l}+\left(\partial_{t}^{2}-L\right) u_{j-2}^{l}\right\}(i \sigma)^{-j+2}=0 \\
& \text { in } \mathbb{R} \times(\Omega \cap U) \quad(l=1, \ldots, d) .
\end{aligned}
$$

Equation (1.20) with $j=0$ means that $\left(I-P_{l}\right) u_{0}^{l}=0$ in $\mathbb{R} \times(\Omega \cap U)$ for $l=1, \ldots, d$. Combining this with (1.18) and (1.19), we have $\sum_{l=1}^{d} P_{l} u_{0}^{l}=0$ and $\sum_{l=1}^{d} \sum_{p, q=1}^{d} v_{p} \varphi_{x_{q}}^{l} a_{p q} P_{l} u_{0}^{l}=0$ on $\mathbb{R} \times(\partial \Omega \cap U)$ respectively. These yield that $P_{l} u_{0}^{l}=0$ on $\mathbb{R} \times(\partial \Omega \cap U)$ for $l=1, \ldots, d$ since the following estimates are derived:

$$
\begin{array}{ll}
\sum_{l=1}^{d}\left|P_{l} v^{l}\right|^{2} \leqq C\left|\sum_{l=1}^{d} P_{l} v^{l}\right|^{2}, . & v^{l} \in \mathbb{R}^{n}, \\
\sum_{l=1}^{d}\left|P_{l} v^{l}\right|^{2} \leqq C\left|\sum_{l=1}^{d} \sum_{p, q=1}^{d} v_{p} \varphi_{x_{q}}^{l} a_{p q} P_{l} v^{l}\right|^{2}, & v^{l} \in \mathbb{R}^{n},
\end{array}
$$

which are seen from the facts that $P_{l}\left(=P_{l}\left(\varphi_{x}^{l}\right)\right)$ is near $P_{l}(v)$ (cf. (1.2)) and that $P_{1}(v), \ldots, P_{d}(v)$ are the orthogonal projections. Applying $P_{l}$ to (1.20) with $j=1$, we get $P_{l}\left(2 \partial_{t}+H\right) P_{l} u_{0}^{l}=0$ in $\mathbb{R} \times(\Omega \cap U)$. Therefore, considering Eq. (1.8) (or (1.11)) as we did for (1.3), we obtain $P_{l} u_{0}^{l}=0$ in $\mathbb{R} \times(\Omega \cap U)$; hence $u_{0}^{l}=0$ in $\mathbb{R} \times(\Omega \cap U)$.

Multiplying (1.20) (with $j=1)$ by $\left(I-P_{l}\right)$ yields that $\left(I-\sum a_{p q} \varphi_{x_{p}}^{l} \varphi_{x_{q}}^{l}\right)\left(I-P_{l}\right) u_{1}^{l}=0$ 
in $\mathbb{R} \times(\Omega \cap U)$, which implies that $\left(I-P_{l}\right) u_{1}^{l}=0$ in $\mathbb{R} \times(\Omega \cap U)$. By the same procedures as for $u_{0}^{l}$, we have $u_{1}^{l}=0$ in $\mathbb{R} \times(\Omega \cap U)$. Continuing these processes inductively, we obtain $u_{j}^{l}=0$ in $\mathbb{R} \times(\Omega \cap U)$ for all $j=0,1, \ldots$ and $l=1, \ldots, d$, which proves the theorem.

\section{Reflected Waves for Plane Incident Waves}

In this section, we assume that $a_{i j}$ are constant matrices satisfying (A.1), (A.2) and (A.3) stated in the introduction and that $L=\sum_{i, j=1}^{n} a_{i j} \partial x_{i} \partial x_{j}$. Furthermore we suppose that the boundary operator is of the form $B u=\left.u\right|_{\partial \Omega}$ or $=\left.N u\right|_{\partial \Omega}$ $\left(N=\sum_{i, j=1}^{n} v_{i} a_{i j} \partial_{x_{j}}\right)$. We use the same notations that in Sect. 1. When they do not depend on the variable $x$, they are abbreviated in the following way: $\lambda_{k}(\xi)\left(=\lambda_{k}(x, \xi)\right)$, $P_{k}(\xi)\left(=P_{k}(x, \xi)\right)$, etc.

If $v_{0} \in \mathbb{R}^{n}$ satisfies $v_{0}=P_{k}(\omega) v_{0}\left(\omega \in S^{n-1}\right)$, the function

$$
v(t, x ; \sigma)=e^{i \sigma\left(\lambda_{k}(\omega)^{-1 / 2} \omega \cdot x-t\right)} v_{0}
$$

is one of the solutions of the equation $\left(\partial_{t}^{2}-L\right) v=0$ (where $\sigma$ is a real parameter with $|\sigma| \geqq 1$ ). We call the solutions of this type the plane waves of $\lambda_{k}$-mode proceeding in the direction $\omega$. Hereafter we fix the $k$ and the $\omega$ arbitrarily. Set

$$
M=\{x \in \partial \Omega: v(x)=\omega\},
$$

and take a sufficiently small neighborhood $U$ of $M$ so that the discussions later are correct.

Since it can be assumed that the gradient of $\varphi\left(x^{\prime}\right)=\lambda_{k}(\omega)^{-1 / 2} \omega \cdot x^{\prime}$ is small enough on $U \cap \partial \Omega$, for each $l(=1, \ldots, d)$ there exists the solution $\varphi^{l}(x)$ of $(1.1)$ with $\varphi\left(x^{\prime}\right)=\lambda_{k}(\omega)^{-1 / 2} \omega \cdot x^{\prime}$. By the procedures stated in Sect. 1, we can construct an asymptotic solution $u(t, x ; \sigma)=\sum_{l=1}^{d} u^{l}(t, x ; \sigma)=\sum_{l=1}^{d}\left\{\sum_{j=0}^{\infty} e^{i \sigma\left(\varphi^{l}(x)-t\right)} u_{j}^{l}(x)(i \sigma)^{-j}\right\}$ such
that

$$
\left\{\begin{array}{lll}
\left(\partial_{t}^{2}-L\right)(u+v) \cong 0 & \text { in } & \mathbb{R} \times(\Omega \cap U) \\
B(u+v) \cong 0 & \text { on } & \mathbb{R} \times(\partial \Omega \cap U)
\end{array}\right.
$$

Note that in this case the amplitude functions $u_{j}^{l}(x)$ do not depend on $t$, and that $u_{j}^{l}$ are determined uniquely (cf. Theorem 1.2).

We choose local coordinates $x^{\prime}(y)$ of $\partial \Omega \cap U$ defined on $\tilde{U} \subset \mathbb{R}^{n-1}(\partial \Omega \cap U=$ $\left.\left\{x^{\prime}(y): y \in \tilde{U}\right\}\right)$. Let $\left(q^{l}(s ; y), p^{l}(s ; y)\right)$ be the bicharacteristic curve associated with $\varphi^{l}(x)$ (defined by (1.7)). With these notations we have

Theorem 2.1. (i) Every principal amplitude function $u_{0}^{l}$ of $u^{l}$ with $l \neq k$ satisfies

$$
u_{0}^{l}\left(q^{l}(s ; y)\right)=0 \quad \text { when } \quad x^{\prime}(y) \in M \quad \text { and } \quad q^{l}(s ; y) \in \Omega \cap U .
$$

(ii) If the derivatives of $v\left(x^{\prime}\right)$ vanishes of order $m+1(\geqq 1)$ on $M$ (i.e. $\partial_{y}^{\alpha} v\left(x^{\prime}(y)\right)=0$ when $1 \leqq|\alpha| \leqq m+1$ and $\left.x^{\prime}(y) \in M\right)$, then the $u_{j}^{l}(x)$ with $l \neq k$ satisfies

$$
\partial_{x}^{\alpha} u_{j}^{l}\left(q^{l}(s ; y)\right)=0 \quad \text { when } \quad|\alpha| \leqq m+1-2 j, x^{\prime}(y) \in M \quad \text { and } \quad q^{l}(s ; y) \in \Omega \cap U
$$


From the above theorem, the following corollary follows immediately:

Corollary. If $\partial \Omega$ is flat on $M$ (i.e. $\partial_{y}^{\alpha} v\left(x^{\prime}(y)\right)=0$ for every $\alpha \neq 0$ when $\left.x^{\prime}(y) \in M\right)$, then $u^{l}(t, x ; \sigma)=O\left(|\sigma|^{-\infty}\right)$ on $\left\{x=q^{l}(s ; y): q^{l}(s ; y) \in \Omega \cap U, x^{\prime}(y) \in M\right\}$ for $l \neq k$.

In the above corollary, if $\partial \Omega$ is not flat on $M$, the conclusion is not necessarily correct. Let us give an example showing it. Let $a_{i j}$ be isotropic (i.e. $a_{i p j q}=$ $\mu\left(\delta_{p q} \delta_{i j}+\delta_{i q} \delta_{j p}\right)+\lambda \delta_{i q} \delta_{j p}, \lambda+\frac{2}{n} \mu>0$ and $\left.\mu>0\right)$. Then, all the assumptions (A.1) (A.3) are satisfied, and the eigenvalues $\left\{\lambda_{l}(\xi)\right\}_{l=1, \ldots, d}$ become of the forms: $\lambda_{1}(\xi)=\mu|\xi|^{2}$ and $\lambda_{2}(\xi)=(\lambda+2 \mu)|\xi|^{2}(d=2)$. Let $B u=\left.u\right|_{\partial \Omega}$, and assume that $\boldsymbol{\Omega}=\left\{x={ }^{t}(y, z) \in \mathbb{R}^{n-1} \times \mathbb{R} ; \psi(y)<z\right\}$. We suppose that $\psi(0)=0, \psi_{y}(0)=0$ and $\psi_{y}(y) \neq 0$ for all $y \neq 0$ in a neighborhood of $y=0$, and consider Eq. (2.1) near the origin. In these situations, we obtain

Remark 2.2. (i) Assume that the Gaussian curvature of $\partial \Omega$ does not vanish at the origin, and choose the incident wave $v(t, x ; \sigma)=e^{i \sigma\left(\lambda_{k}(\omega)^{-1 / 2} \omega \cdot x-t\right)} v_{0}$ as follows:

$$
\omega={ }^{t}(0, \ldots, 0,-1), k=1 \text { and } v_{0}={ }^{t}\left(v_{01}, \ldots, v_{0 n-1}, 0\right) \neq 0
$$
(i.e. $v$ is of $\lambda_{1}(\omega)$-mode). Then the $\lambda_{2}\left(\varphi_{x}^{2}\right)$-mode part $u^{2}(t, x ; \sigma)=\sum_{j=0}^{\infty} e^{i \sigma\left(\varphi^{2}(x)-t\right)} u_{j}^{2}(x)(i \sigma)^{-j}$
satisfies

$$
u_{1}^{2}(0) \neq 0 \text {. }
$$

(ii) Assume that the mean curvature of $\partial \Omega$ does not vanish at the origin, and choose the incident wave as follows:

$$
\omega={ }^{t}(0, \ldots, 0,-1), \quad k=2 \text { and } v_{0}={ }^{t}\left(0, \ldots, 0, v_{0 n}\right) \neq 0
$$

(i.e. $v$ is of $\lambda_{2}(\omega)$-mode). Then the function $u_{1}^{1}(x)$ of the $u^{1}(t, x ; \sigma)$ satisfies

$$
u_{1}^{1}(0) \neq 0 \text {. }
$$

Let us note in the above remark that the incident wave hits $\partial \Omega$ perpendicularly only at $x=0$ (i.e. $M=\{0\}$ ), and that the properties $u_{1}^{l}\left(q^{l}(s ; 0)\right) \neq 0(l=1,2)$ follow for any small $s>0$ in the respective cases. The above remark will be provided at the end of this section.

From now on, we shall prove Theorem 2.1. At first let us check the statement (i). As was discussed in Sect. $1,\left(I-P_{l}\right) u_{0}^{l}=0$, and $P_{l} u_{0}^{l}$ satisfies

$$
\begin{aligned}
& P_{l} H P_{l} u_{0}^{l}=0 \text { in } \Omega \cap U, \\
& P_{l} u_{0}^{l}=\left\{\begin{array}{lllll}
-P_{l} P^{-1} v_{0} & \text { on } & \partial \Omega \cap U & \text { when } & B u=\left.u\right|_{\partial \Omega}, \\
-P_{l} Q^{-1} v_{-1} & \text { on } & \partial \Omega \cap U & \text { when } & B u=\left.N u\right|_{\partial \Omega},
\end{array}\right.
\end{aligned}
$$

where $v_{-1}=\lambda_{k}(\omega)^{-1 / 2} \sum_{i, j=1}^{n} a_{i j} v_{i} \omega_{j} v_{0}$. Furthermore, this is equivalent to the following equation for $w_{0}(s, y)={ }^{t} e\left(q^{l}(s ; y)\right) u_{0}^{l}\left(q^{l}(s ; y)\right)(e(x)$ being the matrix in (1.6)):

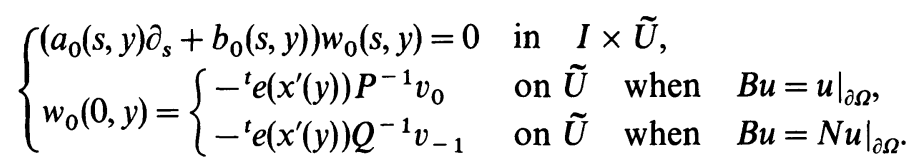


We have $P^{-1} v_{0}=\left\{\sum_{l=1}^{d} P_{l}(w)\right\}^{-1} v_{0}=v_{0}$ and $Q^{-1} v_{-1}=\left\{-\sum_{l=1}^{d} \lambda_{l}(\omega)^{1 / 2} P_{l}(\omega)\right\}^{-1} \times$ $\lambda_{k}(\omega)^{1 / 2} v_{0}=-v_{0}$ when $x^{\prime}(y) \in M$. Therefore, if $l \neq k$, the data $-{ }^{t} e\left(x^{\prime}(y)\right) P^{-1} v_{0}$ and ${ }^{t} e\left(x^{\prime}(y)\right) Q^{-1} v_{-1}$ vanish when $x^{\prime}(y) \in M$. Hence, for these $y$ and $l$ we have $w_{0}(s, y)=0$ on $0 \leqq s \leqq s_{0}$ (by the uniqueness of the solutions of (2.2)), which yields that $P_{l}$ $u_{0}^{l}\left(q^{l}(s ; y)\right)=0$ when $l \neq k$ and $x^{\prime}(y) \in M$. Thus (i) is proved.

Next let us show (ii) in the theorem. To begin with, we note

Lemma 2.3. Assume that

$$
\partial_{y}^{\alpha} v\left(x^{\prime}(y)\right)=0 \quad \text { when } \quad 1 \leqq|\alpha| \leqq m+1(m \geqq 0) \text { and } x^{\prime}(y) \in M .
$$

Then the function $\varphi\left(x^{\prime}\right)=\lambda_{k}(\omega)^{-1 / 2} \omega \cdot x^{\prime}\left(x^{\prime} \in \partial \Omega \cap U\right)$ satisfies

$$
\partial_{y}^{\alpha} \varphi\left(x^{\prime}(y)\right)=0 \text { when } 1 \leqq|\alpha| \leqq m+2 \text { and } x^{\prime}(y) \in M .
$$

We choose a system of orthonormal coordinates ${ }^{t}(y, z)$ in $\mathbb{R}^{n}$ such that $z=-\omega \cdot x$ and that the origin $x=0$ is represented by $z=0, y=0$. Note that correctness of Lemma 2.3 does not depend on choice of the coordinates $x^{\prime}(y)$. Let $\Omega \cap U$ be expressed by $z>\psi(y)$. Then $x^{\prime}(y)={ }^{t}(y, \psi(y))$ become local coordinates of $\partial \Omega \cap U$. It is obvious that

$$
\begin{aligned}
& \omega \cdot x^{\prime}(y)=-\psi(y), \\
& v\left(x^{\prime}(y)\right)=\left(1+\left|\psi_{y}(y)\right|^{2}\right)^{-1 / 2 t}\left(\psi_{y}(y),-1\right) .
\end{aligned}
$$

From these equalities, Lemma 2.3 follows immediately. Hereafter, we introduce these coordinates ${ }^{t}(y, z)$.

Let $\tau_{l}(y, \eta)$ be the function $\tau_{l}$ in (1.2) represented by the variables $(y, \eta)\left(\eta\left(\in \mathbb{R}^{n-1}\right)\right.$ moving near 0$)$ :

$$
\lambda_{l}\left(\tau_{l}(y, \eta) v\left(x^{\prime}(y)\right)+\eta \cdot \partial_{y} x^{\prime}(y)\right)=1 \quad\left(\eta \cdot \partial_{y}=\sum_{i=1}^{n-1} \eta_{i} \partial_{y_{l}}\right), \quad \tau_{l}(y, \eta)<0,
$$

Lemma 2.4. Let $\tau_{l}(y, \eta)$ be the function in (2.4). If $v\left(x^{\prime}(y)\right)$ satisfies

$$
\partial_{y}^{\alpha} v\left(x^{\prime}(y)\right)=0 \quad \text { when } 1 \leqq|\alpha| \leqq m+1(m \geqq 0) \text { and } x^{\prime}(y) \in M \text {, }
$$

then we have for any $\beta$

$$
\partial_{y}^{\alpha} \partial_{\eta}^{\beta} \tau_{l}(y, \eta)=0 \quad \text { when } \quad 1 \leqq|\alpha| \leqq m+1 \quad \text { and } \quad x^{\prime}(y) \in M
$$

Proof. From (2.3), the assumption for $v\left(x^{\prime}(y)\right)$ is equivalent to

$$
\partial_{y}^{\alpha} \psi(y)=0 \text { when } \quad 1 \leqq|\alpha| \leqq m+2 \quad \text { and } \quad x^{\prime}(y) \in M .
$$

Therefore we have

$$
\partial_{y}^{\alpha} x^{\prime}(y)={ }^{t}\left(0, \partial_{y}^{\alpha} \psi(y)\right)=0 \quad \text { when } \quad 2 \leqq|\alpha| \leqq m+2 \quad \text { and } \quad x^{\prime}(y) \in M
$$

Applying $\partial_{y_{i}}$ to Eq. (2.4) yields that $\partial_{\xi} \lambda_{l} \cdot\left(\left(\partial_{y_{i}} \tau_{l}\right) v+\tau_{l} \partial_{y_{1}} v+\eta \cdot \partial_{y} \partial_{y_{i}} x^{\prime}\right)=0$. Therefore it follows that $\left(\partial_{\xi} \lambda_{l} \cdot v\right) \partial_{y_{i}} \tau_{l}=0$ when $x^{\prime}(y) \in M$. Since $\partial_{\xi} \lambda_{l} \cdot v \neq 0$ (assuming that $|\eta|$ is small enough), we obtain $\partial_{y_{i}} \tau_{l}(y, \eta)=0$ when $x^{\prime}(y) \in M$. Repeating this procedure inductively, we get the lemma. The proof is complete.

Lemma 2.5. Let $\varphi\left(x^{\prime}\right)$ and $\varphi^{l}(x)$ be the functions in (1.1), and assume that

$$
\begin{aligned}
& \partial_{y}^{\alpha} \varphi\left(x^{\prime}(y)\right)=0 \text { when } 2 \leqq|\alpha| \leqq m+2 \text { and } x^{\prime}(y) \in M \text {, } \\
& \partial_{y}^{\alpha} v\left(x^{\prime}(y)\right)=0 \quad \text { when } \quad 1 \leqq|\alpha| \leqq m+1 \text { and } x^{\prime}(y) \in M \text {. }
\end{aligned}
$$


Then the projections $P_{l}(\xi)(l=1, \ldots, d)$ satisfy

$\partial_{y}^{\alpha}\left\{P_{l}\left(\varphi_{x}^{l}\left(q^{l}(s ; y)\right)\right)\right\}=0 \quad$ when $\quad 1 \leqq|\alpha| \leqq m+1, \quad q^{l}(s ; y) \in \Omega \cap U$ and $x^{\prime}(y) \in M$.

Proof. Let us note that $\varphi_{x}^{l}\left(q^{l}(s ; y)\right)=p^{l}(s ; y)=p^{l}(0 ; y)$ since $\partial_{x} \lambda_{l}=0$. This yields that

$$
p^{l}(s ; y)=\tau_{l}\left(y, A^{-1} \varphi_{y}\right) v+\left(A^{-1} \varphi_{y}\right) \cdot \partial_{y} x^{\prime}(y)
$$

where $A=A(y)$ is the $(n-1) \times(n-1)$ matrix whose $(i, j)$-component is $x_{y_{i}}^{\prime} \cdot x_{y_{j}}^{\prime}=$ $\delta_{i j}+\psi_{y_{i}}(y) \psi_{y_{j}}(y)$. We see that $\partial_{y}^{\alpha} A=0$ when $1 \leqq|\alpha| \leqq 2 m+2$ and $x^{\prime}(y) \in M$; therefore, $\partial_{y}^{\alpha} A^{-1}=0$ when $1 \leqq|\alpha| \leqq 2 m+2$ and $x^{\prime}(y) \in M$. Hence, applying $\partial_{y}^{\alpha}$ to the equality (2.5), by Lemma 2.4 we have

$\partial_{y}^{\alpha} p^{l}(s ; y)=0 \quad$ when $\quad 1 \leqq|\alpha| \leqq m+1, \quad q^{l}(s, y) \in \Omega \cap U \quad$ and $\quad x^{\prime}(y) \in M$, which proves the lemma.

From the construction of $e$ in (1.6), it follows that $e\left(q^{l}(s ; y)\right)$ does not depend on $s$; we denote it by $e(y)$. Furthermore, by Lemma 2.5 , this $e(y)$ satisfies

$$
\partial_{y}^{\alpha} e(y)=0 \text { when } 1 \leqq|\alpha| \leqq m+1 \quad \text { and } \quad x^{\prime}(y) \in M,
$$

if $\partial_{y}^{\alpha} \varphi\left(x^{\prime}(y)\right)=0$ when $2 \leqq|\alpha| \leqq m+2$ and $x^{\prime}(y) \in M$ and $\partial_{y}^{\alpha} v\left(x^{\prime}(y)\right)=0$ when $1 \leqq|\alpha| \leqq$ $m+1$ and $x^{\prime}(y) \in M$. Let us note that the first condition ' $\partial_{y}^{\alpha} \varphi\left(x^{\prime}(y)\right)=0$ ' follows from the second one ' $\partial_{y}^{\alpha} v\left(x^{\prime}(y)\right)=0$ ' if $\varphi\left(x^{\prime}\right)=\lambda_{k}(\omega)^{-1 / 2} \omega \cdot x^{\prime}$ (cf. Lemma 2.3). Hereafter we put $\varphi\left(x^{\prime}\right)=\lambda_{k}(\omega)^{-1 / 2} \omega \cdot x^{\prime}$, and assume that $\partial_{y}^{\alpha} v\left(x^{\prime}(y)\right)=0$ when $1 \leqq|\alpha| \leqq m+1$ and $x^{\prime}(y) \in M$.

As was shown in the proof of Lemma 2.5, the following equality holds:

$\partial_{y}^{\alpha}\left\{\varphi_{x}^{l}\left(q^{l}(s ; y)\right)\right\}=\partial_{y}^{\alpha} p^{l}(0 ; y)=0 \quad$ when $\quad 1 \leqq|\alpha| \leqq m+1 \quad$ and $\quad x^{\prime}(y) \in M$.

In view of the definition of $\left(q^{l}, p^{l}\right)$ (cf. (1.7)), we see that $\partial_{s}^{\beta} \partial_{y}^{\alpha}\left(\partial_{s} q^{l}, \partial_{y_{1}} q^{l}, \ldots, \partial_{y_{n-1}} q^{l}\right)(s$, $y)=0$ when either ' $\beta \geqq 2$ ' or ' $1 \leqq \beta+|\alpha|,|\alpha| \leqq m$ and $x^{\prime}(y) \in M$ ', which means that

$$
\begin{aligned}
\partial_{s}^{\beta} \partial_{y}^{\alpha} \frac{\partial s}{\partial x_{i}}(s, y)=\partial_{s}^{\beta} \partial_{y}^{\alpha} \frac{\partial y_{j}}{\partial x_{i}}(s, y)=0 \quad \text { if either ' } \beta \geqq 2 \text { ' } \\
\text { or ' } 1 \leqq \beta+|\alpha|,|\alpha| \leqq m \text { and } x^{\prime}(y) \in M \text { '. }
\end{aligned}
$$

Mixing (2.6), (2.7) and (2.8) yields that

$$
\begin{aligned}
& \partial_{s}^{\beta} \partial_{y}^{\alpha} a_{i}(s, y)=0 \quad(i=0, \ldots, n-1) \quad \text { when } \quad 1 \leqq|\alpha| \leqq m \quad \text { and } \quad x^{\prime}(y) \in M \text {, } \\
& \partial_{s}^{\beta} \partial_{y}^{\alpha} b_{0}(s, y)=0 \text { for } \beta \geqq 0 \quad \text { when } 0 \leqq|\alpha| \leqq m \text { and } x^{\prime}(y) \in M \text {. }
\end{aligned}
$$

As was seen in Sect. $1, w_{j}(s, y)={ }^{t} e u_{j}^{l}\left(q^{l}(s ; y)\right)$ satisfies

$$
\begin{cases}\left(a_{0}(s, y) \partial_{s}+b_{0}(s, y)\right) w_{j}(s, y)=f_{j}(s, y) & \text { in } I \times \tilde{U} \\ w_{j}(0, y)=g_{j}(y) & \text { on } \tilde{U}\end{cases}
$$

where $f_{j}=-{ }^{t} e\left\{H\left(I-P_{l}\right) u_{j}^{l}+L u_{j-1}^{l}\right\}, g_{j}={ }^{t} e P^{-1}\left(-\sum_{i=1}^{d}\left(I-P_{i}\right) u_{j}^{i}-\delta_{0 j} v_{0}\right)$ when $B u=\left.u\right|_{\partial \Omega}$ and $g_{j}={ }^{t} e Q^{-1}\left(-\sum_{i=1}^{d}\left(N \varphi^{i}\right)\left(I-P_{i}\right) u_{j}^{i}-\sum_{i=1}^{d} N u_{j-1}^{i}-\delta_{0 j} v_{-1}\right) \quad$ when $B u=\left.N u\right|_{\partial \Omega}$. Note that $a_{0}-I=b_{0}=0$ when $x^{\prime}(y) \in M$ (cf. Lemma 1.4 and (2.9)); furthermore, $g_{0}=0$ when $l \neq k$ and $x^{\prime}(y) \in M$, and $f_{0}=0$. Therefore it is seen from (2.10) that $w_{0}(s, y)=\partial_{s} w_{0}(s, y)=0$ when $x^{\prime}(y) \in M$ and $l \neq k$. From the definitions of $P(y)\left(=P\left(x^{\prime}(y)\right)\right), Q(y)\left(=Q\left(x^{\prime}(y)\right)\right.$ and Lemma 2.5 , we have $\partial_{y}^{\alpha} P(y)=\partial_{y}^{\alpha} Q(y)=0$ when 
$1 \leqq|\alpha| \leqq m+1$ and $x^{\prime}(y) \in M$, which yields that

$$
\partial_{y}^{\alpha} P^{-1}(y)=\partial_{y}^{\alpha} Q^{-1}(y)=0 \quad \text { when } \quad 1 \leqq|\alpha| \leqq m+1 \quad \text { and } \quad x^{\prime}(y) \in M .
$$

Combining this with (2.6) and Lemma 2.5, we obtain

$$
\partial_{y}^{\alpha} g_{0}(s, y)=0 \text { when } 1 \leqq|\alpha| \leqq m+1 \quad \text { and } \quad x^{\prime}(y) \in M .
$$

Differentiating Eq. (2.10) (with $j=0)$ in $y_{i}$ and fixing $y$ so that $x^{\prime}(y) \in M$, by (2.9) and (2.11) we have $\partial_{y_{i}} w_{0}(s, y)=0$ for $i=1, \ldots, n-1$ if $l \neq k$. Repeating this procedure yields that for any $\beta=1,2, \ldots$,

$$
\partial_{s}^{\beta} \partial_{y}^{\alpha} w_{0}(s, y)=0 \text { when } \quad l \neq k, 1 \leqq|\alpha| \leqq m+1, x^{\prime}(y) \in M .
$$

Therefore we obtain the statement (ii) in Theorem 2.1 for $j=0$.

In the same way, noting that $\partial_{s}^{\beta} \partial_{y}^{\alpha} f_{j}$ and $\partial_{y}^{\alpha} g_{j}=0$ for $|\alpha| \leqq m+1-2 j$ if $\partial_{s}^{\beta} \partial_{y}^{\alpha} u_{j-1}^{l}=0$ for $|\alpha| \leqq m+3-2 j$ and any $\beta$, by induction we get (ii) in the theorem for $j \geqq 1$. The proof is complete.

Proof of Remark 2.2. Let us prove statement (i). For $\xi, \eta \in \mathbb{R}^{n}$ we denote the matrix by $\xi \otimes \eta$ whose $(i, j)$-component is $\xi_{i} \eta_{j}$. It is seen that $H$ and $P_{2}(\xi)$ are of the forms

$$
\begin{aligned}
& H=2 \mu\left(\varphi_{x}^{2} \cdot \partial_{x}\right) I+(\mu+\lambda) \varphi_{x}^{2} \otimes \partial_{x}+(\mu+\lambda) \partial_{x} \otimes \varphi_{x}^{2}+L \varphi^{2}, \\
& P_{2}(\xi)=\left(|\xi|^{-1} \xi\right) \otimes\left(|\xi|^{-1} \xi\right),
\end{aligned}
$$

where the $(i, j)$-component of $\partial_{x} \otimes \varphi_{x}^{2}$ means $\varphi_{x_{j}}^{2} \partial_{x_{i}}$. The equations of $q^{2}(s ; y)$ and $p^{2}(s ; y)\left(=\varphi_{x}^{2}\left(q^{2}(s ; y)\right)(\mathrm{cf} .(1.7))\right.$ become of the forms

$$
\left\{\begin{array}{l}
\frac{d q^{2}}{d s}=2(\lambda+2 \mu) p^{2}, \quad \frac{d p^{2}}{d s}=0, \\
q^{2}(0 ; y)={ }^{t}(y, \psi(y)), \quad p^{2}(0 ; y)=\varphi_{x}^{2}(y, \psi(y)) .
\end{array}\right.
$$

From the equalities that $\varphi^{2}(y, \psi(y))=-\mu^{-1 / 2} \psi(y)$ and $\left|\varphi_{x}^{2}\right|^{2}=(\lambda+2 \mu)^{-1}$, it is seen that

$$
\begin{aligned}
\varphi_{x}^{2}(y, \psi(y))= & -\mu^{-1 / 2} \sum_{i=1}^{n-1}\left(A^{-1} \psi_{y}\right)_{i}^{t}\left(0, \ldots, \stackrel{i}{1}, \ldots, 0, \psi_{y_{i}}\right) \\
& +\left\{(\lambda+2 \mu)^{-1}-\mu^{-1 t} \psi_{y} A^{-1} \psi_{y}\right\}^{1 / 2}\left(1+\left|\psi_{x}\right|^{2}\right)^{-1 / 2 t}\left(-{ }^{t} \psi_{y}, 1\right)
\end{aligned}
$$

where $A$ is the matrix in $(2.5)$ and $\left(A^{-1} \psi_{y}\right)_{i}$ means $i$-component of the vector $A^{-1} \psi_{y}$. Note that $A-I=\partial_{y_{i}} A=0(i=1, \ldots, n-1)$ when $y=0$; hence, $A^{-1}-I=\partial_{y_{i}} A^{-1}=0$ $(i=1, \ldots, n-1)$ when $y=0$.

Take the coordinates $y$ so that $\partial_{y_{i}} \partial_{y_{j}} \psi(0)=\delta_{i j} \partial_{y_{i}}^{2} \psi(0)$. Then, (2.13) yields that

$$
\begin{aligned}
\varphi_{x}^{2}(0)=(\lambda+2 \mu)^{-1 / 2 t}(0,1), & \\
\left.\partial_{y_{i}}\left\{\varphi_{x}^{2}(y, \psi(y))\right\}\right|_{y=0}= & -\left(\mu^{-1 / 2}+(\lambda+2 \mu)^{-1 / 2}\right) \psi_{y_{i} y_{i}}(0) \\
& { }^{t}(0, \ldots, 1, \ldots, 0) \quad(i=1, \ldots, n-1) .
\end{aligned}
$$

Therefore, in view of (2.12) we have

$$
\begin{aligned}
& P_{2}\left(\varphi_{x}^{2}(0)\right)=K_{n}, \\
& \left.\partial_{y_{i}} P_{2}\left(\varphi_{x}^{2}(y, \psi(y))\right)\right|_{y=0}=-\left(\mu^{-1 / 2}+(\lambda+2 \mu)^{-1 / 2}\right) \psi_{y_{i} y_{i}}(0) K_{i} \\
& (i=1, \ldots, n-1),
\end{aligned}
$$




$$
\begin{aligned}
& \text { where } K_{i}=\left(\begin{array}{ccccc} 
& & & & 0 \\
& & & & \vdots \\
& & & & \vdots \\
0 & \cdots & 1 & \cdots & 0
\end{array}\right)^{i}(i=1, \ldots, n) \text {. In the same way we get } \\
& P_{2}\left(\varphi_{x}^{1}(0)\right)=K_{n} \text {, } \\
& \left.\partial_{y_{i}} P_{2}\left(\varphi_{x}^{1}(y, \psi(y))\right)\right|_{y=0}=-2 \mu^{-1 / 2} \psi_{y_{i} y_{i}}(0) K_{i} \quad(i=1, \ldots, n-1) .
\end{aligned}
$$

Differentiating the equality $P(y) P(y)^{-1}=I\left(P(y)=P_{1}\left(\varphi_{x}^{1}(y, \psi(y))+P_{2}\left(\varphi_{x}^{2}(y, \psi(y))\right)\right)\right.$, and using (2.14) and (2.15), we have

$$
\partial_{y_{i}} P^{-1}(0)=\left((\lambda+2 \mu)^{-1 / 2}-\mu^{-1 / 2}\right) \psi_{y_{i} y_{i}}(0) K_{i} \quad(i=1, \ldots, n-1) .
$$

Since $e(y)=(\lambda+2 \mu)^{1 / 2} \varphi_{x}^{2}(y, \psi(y))$, it follows from $(2.13)$ that $e(0)={ }^{t}(0, \ldots, 0,1)$. Therefore, noting that $v_{0}={ }^{t}\left(v_{10}, \ldots, v_{0 n-1}, 0\right)$ and $P(0)^{-1}=1$, from Eq. (2.2) of $w_{0}$ we obtain $w_{0}(s, 0)=0$ for any $s$. This yields that

$$
u_{0}^{2}(0)=\partial_{x_{n}} u_{0}^{2}(0)=0
$$

since $\quad u_{0}^{2}\left(q^{2}(s ; y)\right)=e(y) w_{0}(s, y) \quad$ and $\quad \partial_{s}\left\{u_{0}^{2}\left(q^{2}\right)\right\}=\sum_{i=1}^{n-1} u_{0 x_{i}}^{2} \frac{d q_{i}^{2}}{d s}+u_{0 x_{n}}^{2} \frac{d q_{n}^{2}}{d s}=$ $(\lambda+2 \mu)^{-1} u_{0 x_{n}}^{2}(0)$ for $y=0$. Furthermore, applying $\partial_{y_{i}}$ to $u_{0}^{2}(y, \psi(y))\left(=P_{2} u_{0}^{2}\right)=$ $-P_{2}\left(\varphi_{x}^{2}(y, \psi(y)) P(y)^{-1} v_{0}\right.$ (cf. (1.4)) and using (2.15) and (2.16), we have

$$
\partial_{x_{i}} u_{0}^{2}(0)=2 \mu^{-1 / 2} \psi_{y_{i} y_{i}}(0) v_{0 i}{ }^{t}(0, \ldots, 0,1) \quad(i=1, \ldots, n-1)
$$

From (1.5) it follows that $\left(I-P_{2}\right) u_{1}^{2}=\left(1-\lambda_{1}\left(\varphi_{x}^{2}\right)\right)^{-1}\left(I-P_{2}\right) H u_{0}^{2}$. It is seen that

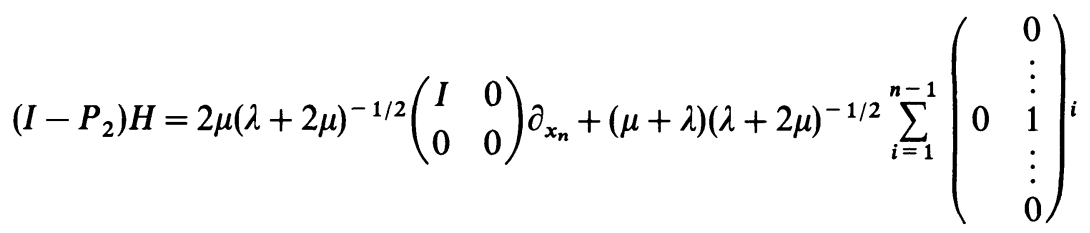

$\partial_{x_{i}}+\left(\begin{array}{ll}I & 0 \\ 0 & 0\end{array}\right) L \varphi^{2}$ when $x=0$. Therefore, by (2.17) and (2.18), we obtain

$$
\left(I-P_{2}\right) u_{1}^{2}(0)=2 \mu^{-1 / 2}(\lambda+2 \mu)^{1 / 2 t}\left(v_{01} \psi_{y_{1} y_{1}}(0), \ldots, v_{0 n-1} \psi_{y_{n-1} y_{n-1}}(0), 0\right)
$$

The above equality shows that $\left(I-P_{2}\right) u_{1}^{2}(0) \neq 0$ since at least one of $v_{0 i} \psi_{y_{i} y_{i}}(0)$ $(i=1, \ldots, n-1)$ does not vanish from the assumptions.

Next, let us consider the statement (ii). In the same way as in the case of (i), we see that

$$
\left(I-P_{1}\right) u_{1}^{1}(0)=\mu^{1 / 2}\left((\lambda+2 \mu)^{-1 / 2}+\mu^{-1 / 2}\right) v_{0 n}\left(\sum_{i=1}^{n-1} \psi_{y_{i} y_{i}}(0)\right) t(0, \ldots, 0,1),
$$

which proves that $u_{1}^{1}(0) \neq 0$. 


\section{References}

1. Friedrichs, K. O.: Symmetric hyperbolic system of linear differential equations. Commun. Pure Appl. Math. 7, 345-392 (1954)

2. Karal, F. C., Keller, J. B.: Elastic wave propagation in homogeneous and inhomogeneous media. J. Acaustic. Soc. Am. 31, 694-705 (1959)

3. Lax, P.: Asymptotic solutions of oscillatory initial value problems. Duke Math. J. 24, 627-646 (1957)

4. Mizohata, S.: The theory of partial differential equations. Cambridge University Press: London 1973

5. Soga, H.: Asymptotic solutions of the elastic wave equation and their applications. Bull. Fac. Educ., Ibaraki Univ. (Nat. Sci.) 38, 9-16 (1989)

Communicated by H. Araki 